\title{
Cognitive Blackouts in Mild Cognitive Impairment and Alzheimer's Dementia
}

\author{
Georg Adler Agnies Marczak Jana Binder \\ Institut für Studien zur Psychischen Gesundheit (ISPG), Mannheim, Germany
}

\section{Keywords}

Mild cognitive impairment · Subjective memory impairment · Alzheimer's dementia .

Self-perception

\begin{abstract}
Background: Cognitive blackouts, e.g. moments of amnesia, disorientation, or perplexity may be an early sign of incipient Alzheimer's dementia (AD). A short questionnaire, the checklist for cognitive blackouts (CCB), was evaluated cross-sectionally in users of a memory clinic. Methods: The CCB was performed in 130 subjects, who further underwent a neuropsychological and clinical examination. Subjective memory impairment and depressive symptoms were assessed. Differences in the CCB score between diagnostic groups and relationships with cognitive performance, depression, and subjective memory impairment were analyzed. $\boldsymbol{R e}$ sults: The CCB score was increased in mild cognitive impairment of the amnestic type or mild $A D$ and correctly predicted $69.2 \%$ of the respective subjects. It was negatively correlated with cognitive performance, positively correlated with depressive symptoms, and substantially increased in subjects who estimated their memory poorer than that of other persons of their age. Discussion: The CCB may be a helpful screening tool for the early recognition of AD.
\end{abstract}

(C) 2018 The Author(s)

Published by S. Karger AG, Basel

\section{Introduction}

In incipient Alzheimer's dementia (AD) there is a continuous deterioration of cognitive capabilities [1]. However, many patients report that long before the onset of dementia, they started to have frightening transient cognitive blackouts, moments of amnesia, disorientation, or perplexity. First, this happened on rare occasions, e.g. if they were short of sleep, under stress or physically ill. In the further course, they experienced that type of occurrences more frequently, within more domains and without obvious reason. 
Table 1. Items of the checklist for cognitive blackouts (CCB)

Within the last six months how often did it happen to you that ...

(1) ... you wanted to get something from another room and when you got there you had forgotten what it was that you went there for?

(2) ... you forgot to keep a date or an appointment or would have forgotten them without being reminded about it beforehand (e.g., by a calendar or by others)?

(3) ... you took a break from reading a book or a text, and when you returned to it you had serious difficulties in recalling what you had read or picking up where you had left off?

(4) ... you had to think about what month it is?

(5) ... you had significant difficulties finding your way at a place that was new to you (e.g., in a hotel or locating your car in a big shopping center's parking lot)?

Thus, before and at the early onset of dementia, there may be a phase characterized by increasingly frequent breakdowns of memory, orientation or executive functions whilst overall cognitive performance is unimpaired most of the time. Consequently, in early stages of dementia, neuropsychological testing may yield inconclusive results and the patients' own appraisal of performance as reflected by subjective memory impairment (SMI) may be more informative [2]. SMI has been found to be related to Alzheimer-like morphological brain changes [3-5], to cerebral amyloid deposition [6, 7], and to the risk of developing dementia [8-10].

If the self-perception of cognitive blackouts in everyday life is the basis for SMI, a detailed assessment of the extent and frequency of these events may be helpful for an early detection of dementia. Based on patients' self-observations, we developed a checklist for cognitive blackouts (CCB) [11]. It has been constructed on the basis of interviews in patients with mild AD. In this study, we present a cross-sectional evaluation of the CCB in users of our memory clinic.

\section{Methods}

The CCB is made up of five items describing frequent self-observations of persons with beginning AD (Table 1).

For items 2-5, the frequency of occurrence of the respective observations is graded as 0 (for "never"), 1 (for "rarely" or "less than once a week"), 2 (for "frequently" or "once or several times a week"), and 3 (for "permanently" or "once or several times a day"). Item 1, the frequency of which had been observed to be inversely related to cognitive impairment [11], is used to compensate for underreporting and is graded in the reverse sense. For each of these items, 0-3 points are issued, leading to CCB scores between 0 and 15 .

The study has been approved by the Institute's committee on human research, and informed consent has been given by the participating subjects. The CCB was evaluated in 130 consecutive users of our memory clinic, 81 women (62.3\%) and 49 men (37.7\%) aged between 50 and 85 years (mean: 62.7 years). Subjects with dementing disorders other than AD, Parkinson syndrome, or a diagnosis of depression were not included.

Assessment of cognitive performance and assignment to the diagnostic groups of "no cognitive impairment" (NCI), "mild cognitive impairment of the amnestic type" (MCIa) and "mild AD" (mAD) were performed by means of the Structured Interview for the Diagnosis of Dementia of the Alzheimer Type, Multi-Infarct Dementia and Dementias of Other Etiology according to ICD-10 and DSM-III-R (SIDAM) [12]. The neuropsychological test battery of the SIDAM includes the Mini-Mental State Examination (MMSE) [13]. If appropriate, further 
clinical, laboratory, or imaging investigations were initiated. Depressive symptoms were examined by means of the German version of the Beck Depression Inventory (BDI-II) [14].

The presence of SMI was assessed by asking the patients: (1) Do you feel like your memory is becoming worse? (2) If so, does it worry you? (3) Do you think that your memory is poorer than that of other persons of your age?

After a normal distribution of the CCB values had been ascertained by a Shapiro-Wilk test, differences in the CCB score between diagnostic groups were examined by a one-way ANOVA and subsequent Student $t$ tests. For the prediction of diagnosis by means of the CCB score, a binary logistic regression analysis was applied using a CCB cutoff score of $\geq 7$. Relationships between CCB score and SIDAM syndromes or scores were studied by means of linear regression analyses using the Pearson correlation coefficient, relationships between CCB score and responses to the SMI questions by Student $t$ tests.

\section{Results}

NCI was found in 67 subjects (51.6\%), MCla in 41 (31.5\%), and mAD in $22(16.9 \%)$.

A one-way ANOVA revealed significant differences in the CCB score between subjects with NCI, MCIa, and mAD $(p<0.001)$. Subsequent $t$ tests showed significant differences between subjects with NCI and MCIa $(p<0.005)$, between subjects with NCI and mAD ( $p<$ 0.001 ), but not between subjects with MCIa and mAD.

The binary logistic regression analysis with the CCB score as independent variable (cutoff $\geq 7$ ) and diagnosis ( $\mathrm{NCI}$ vs. $\mathrm{MCIa} / \mathrm{mAD}$ ) as dependent variable showed a significant improvement of classification by $12.3 \%(p<0.001)$. The positive predictive value of the CCB score for MCIa or mAD was $69.2 \%$, the negative predictive value was $84.8 \%$.

There were significant negative correlations between the CCB score and the SIDAM syndromes and scores. Correlation with the MMSE score was $-0.426(p<0.001)$. Among the SIDAM syndromes, the correlations were highest for "orientation" $(R=-0.432 ; p<0.001)$ and "short-term memory" $(R=-0.386 ; p<0.001)$. The CCB and BDI-II scores were positively correlated $(R=0.249 ; p<0.01)$.

When the subjects were questioned about SMI, the answer to question 1 was affirmative in 86 subjects (66.2\%), to question 2 in 64 (49.2\%), and to question 3 in 41 (31.5\%). The CCB score was significantly increased for an affirmative answer to each of these questions, with the most significant difference for an affirmative answer to question 3 (7.2 \pm 2.6 vs. $5.0 \pm 1.8$; $p<0.001)$.

\section{Discussion}

The CCB score allowed a correct prediction of MCIa or mAD in $69.2 \%$ of the users of an outpatient memory clinic. It was negatively correlated with cognitive performance and positively correlated with depressive symptoms.

With progressing cognitive decline, the awareness of cognitive impairment may decrease [15]. Consequently, people with more profound cognitive impairment have been found to report less SMI [16]. This may hold true, perhaps to a lesser degree, for the self-perception of cognitive blackouts. Thus, in order to at least partially compensate for underreporting, we included item 1 into the CCB, which describes a frequent working memory failure unrelated to dementia. In a preliminary analysis, we indeed found that the respective observation ("you wanted to get something from another room and when you got there you had forgotten what it was that you went there for") was reported less frequently by the subjects with mAD than 
by the cognitively unimpaired ones [11]. Thus, the CCB as well as the assessment of SMI may be better suited to detect mild cognitive impairment than mild or moderate dementia [17].

When differentiating the features of the self-perception of cognitive blackouts and those of SMI, the frame of reference may be relevant. The CCB had the highest correlation with the self-perceived memory impairment when comparing it with other persons. The correlations were not that high for self-perceived memory decline and concerns about it, which tend to refer to a past-self comparison and may rather be influenced by worries and fears. To a certain extent, this may also apply to cognitive blackouts, as we found a positive correlation between the CCB score and depressive symptoms. However, the more objectifying approach of the CCB may mitigate the influence of affective involvement.

Thus, the CCB may be a helpful tool for screening purposes and may be readily applied in general medicine settings. In further studies underway, the relationship of the CCB to known risk factors and the course of cognitive performance are examined.

\section{Acknowledgements}

Dr. Yvonne Lembach is gratefully acknowledged for her support in the development of the CCB. Prof. Dr. Angelika Mautes is thanked for critical reading of the manuscript.

\section{Disclosure Statement}

This research did not receive any specific grant from funding agencies in the public, commercial, or not-for-profit sectors. None of the authors has any conflict of interest to declare.

\section{References}

1 Verlinden VJA, van der Geest JN, de Bruijn RFAG, Hofman A, Koudstal PJ, Ikram MA: Trajectories of decline in cognition and daily functioning in preclinical dementia. Alzheimers Dement 2016;12:144-153.

-2 Jessen F, Amariglio RE, van Boxtel M, Breteler M, Ceccaldi M, Chételat G, et al: A conceptual framework for research on subjective cognitive decline in preclinical Alzheimer's disease. Alzheimers Dement 2014;10:844852.

-3 Jessen F, Feyen L, Freyman K, Tepest R, Maier W, Heun R, et al: Volume reduction of the entorhinal cortex in subjective memory impairment. Neurobiol Aging 2006;27:1751-1756.

-4 Tepest R, Wang L, Csernansky JG, Heun R, Scheef L, Jessen F: Hippocampal surface analysis in subjective memory impairment, mild cognitive impairment and Alzheimer's dementia. Dement Geriatr Cogn Disord 2008;26:323-329.

5 Schultz SA, Oh JM, Koscik RL, Dowling NM, Gallagher CL, Carlsson CM, et al: Subjective memory complaints, cortical thinning, and cognitive dysfunction in middle-age adults at risk for AD. Alzheimers Dement 2015;11: 33-40.

6 Amariglio RE, Becker JA, Carmasin J, Wadsworth LP, Lorius N, Sullivan C, et al: Subjective cognitive complaints and amyloid burden in cognitively normal older individuals. Neuropsychologia 2012;50:2880-2886.

-7 Perrotin A, Mormino EC, Madison CM, Hayenga AO, Jagust WJ: Subjective cognition and amyloid deposition imaging: a Pittsburgh Compound B positron emission tomography study in normal elderly individuals. Arch Neurol 2012;69:223-239.

8 Reisberg B, Shulman MB, Torossian C, Leng L, Zhu W: Outcome over seven years of healthy adults with and without subjective cognitive impairment. Alzheimers Dement 2010;6:11-24.

-9 Jessen F, Wiese B, Bachmann C; Eifflaender-Gorfer S, Haller F, Kölsch H, et al: Prediction of dementia by subjective memory impairment: effects of severity and temporal association with cognitive impairment. Arch Gen Psychiatry 2010;67:414-422.

10 Mitchell AJ, Beaumont H, Ferguson D, Yadegarfar M, Stubbs B: Risk of dementia and mild cognitive impairment in older people with subjective memory complaints: meta-analysis. Acta Psychiatr Scand 2014;130:439-451. 
11 Adler G: Zeichen der beginnenden Demenz: Eine Checkliste für kognitive Blackouts. Hamburg, Tredition, 2017.

12 Zaudig M, Hiller W: SIDAM - Strukturiertes Interview für die Diagnose einer Demenz vom Alzheimer Typ, der Multiinfarkt- (oder vaskulären) Demenz und Demenzen anderer Ätiologie nach DSM-III-R, DSM-IV und ICD-10 (SIDAM-Handbuch). Bern, Huber, 1996.

$\checkmark 13$ Folstein MF, Folstein ME, McHugh PR: Mini-Mental State: a practical method for grading the cognitive state of patients for the clinician. J Psychiatr Res 1975;12:189-198.

14 Hautzinger M, Keller F, Kühner C: Beck Depressions-Inventar (BDI-II). Revision. Frankfurt/Main, Harcourt Test Services, 2006.

15 Zamboni G, Wilckock G: Lack of awareness of symptoms in people with dementia: the structural and functional basis. Int J Geriatr Psychiatry 2011;26:783-792.

16 Auw CCK: Association of cognitive impairment and depressive symptoms with subjective memory complaints in people with dementia. Asian J Gerontol Geriatr 2013;8:16-20.

17 Eichler T, Thyrian JR, Hertel J, Wucherer D, Michalowsky B, Reiner K, et al: Subjective memory impairment: no suitable criteria for case-finding of dementia in primary care. Alzheimers Dement 2015;11:179-186. 